\title{
Biochemical and Histochemical Study on Fatty Acid-Calcium Gallstones
}

\author{
Tadao Sanjo \\ Department of Surgery, Tohoku University School of \\ Medicine, Sendai
}

\begin{abstract}
Sanjo, T. Biochemical and Histochemical Study on Fatty Acid-Calcium Gallstones. Tohoku J. exp. Med., 1970, 101 (3), 237-249 — Chemical, mineralogical and histochemical investigations of fatty acid-calcium stones were carried out in order to elucidate the mechanism of formation of gallstone components in general. The results obtained were as follows: 1) Systematic chemical analysis, infrared spectroscopy and gas chromatography of fatty acid-calcium gallstones obtained from 3 patients revealed that the predominant fatty acid in these stones was palmitic acid. Small amounts of stearic acid and oleic acid were also detected. 2) Fatty acidcalcium gallstones showed a laminated structure characterized by eccentrically arranged layers. Examinations under polarized light revealed fatty acid-calcium crystals between the layers of bile pigment particles which extended outwards in a fan-shape. 3) Histochemical examinations revealed the presence of diffuse networks of acid mucopolysaccharides containing sulfated radicals in fatty acid-calcium stones. Detailed examination of the net-works revealed a ubiquitous presence of acid mucopolysaccharides in the spaces between fatty acid-calcium crystals as well as in the layers,suggesting that these substances were playing an important role in holding crystals and particles together as bridges among other components of the stones. 4) On the basis of these findings it was concluded that severe infection of the biliary tract, release of fatty acid-calcium into the bile by enzymes and concrement formation of composite materials by acid mucopolysaccharide are essential in the formation of gallstones of this type.__ fatty acid-calcium gallstone; acid mucopolysaccharide
\end{abstract}

Although gallstones are most commonly composed of cholesterol, bile pigments and their calcium salts, those composed mainly of calcium salt of fatty acid are found in the bile duct on rare occasions. The gallstones of this type were first described by Nishimura 1 in 1938 who reported 3 cases of cholelithiasis in which the concrements were found by chemical analysis to be composed of fatty acid-calcium, especially calcium palmitate. Only a few similar cases have been subsequently reported by Miyake et $a .^{2}$ and Nishimura ${ }^{3,4}$ in Japan, and there has been no report of similar cases from European and American countries. Consequently, there has not been detailed investigation of the pathogenesis of the stone of this type. Reviewing the reported cases and on the basis of our own study, it has been suggested that the fatty acid-calcium stones are formed under contamination of the bile by Escherichia coli in the biliary tract. In dealing with the pathogenesis of the gallstone, Maki ${ }^{5}$ and Maki and Suzuki ${ }^{6}$ emphasized the importance of the following three

Received for publication. February 23, 1970. 
points: 1) the causative factor, 2) the mechanism by which the component of the stone is precipitated, and 3) the mechanism by which the concrement is formed from the precipitated components. The mechanism of formation of calcium bilirubinate and calcium carbonate stones was clarified by the investigation along this line.

In this study, the fatty acid-calcium stones were investigated biochemically, mineralogically and histochemically to elucidate the mechanism of their formation.

\section{Materials}

In three patients with cholelithiasis operated on at our clinic, the gallstones of the above-mentioned type were found. In all the three patients, the stones were found in the common bile duct and intrahepatic bile ducts. The stones of this type were irregular in shape, variable in size and fragile in consistency. The color of their surface varied from dark brown to dark gray. The cut surface was yellowish-white in color with waxy luster and showed multiple non-concentric layers as is shown in Figs. 1, 2 and 3.

\section{Methods}

\section{1) Methods of chemical analysis}

The method of chemical analysis is shown diagrammatically in Fig. 4. The stones were ground and desiceated in vacuo on phosphorus pentoxide. Three hundred to five hundred $\mathrm{mg}$ of desiccated powder thus obtained were put in $50 \mathrm{ml}$ of chloroform and were left at room temperature for $\mathbf{2 4}$ hours. The mixture was then passed through glass filter and the filtrate was marked as Extract 1. About $50 \mathrm{ml}$ of $1 \%$ disodium ethylenediamine tetraacetate were added to the residue. After 12 hours standing at room temperature the mixture was filtered and the filtrate was marked as Extract 2. The residue was desiccated in vacuo on phosphorus pentoxide again and the procedure of extraction with chloroform was repeated. The extract thus obtained was marked as Extract 3. About $50 \mathrm{ml}$ of $1 \% \mathrm{HCl}$ were added to the insoluble residue and the mixture was left at room temperature for 12 hours. Filtration of the mixture yielded Extract 4. The residue was left in chloroform for 12 to 24 hours at $37^{\circ} \mathrm{C}$, and Extract 5 and the final residue were obtained. Contents of fatty acid, cholesterol, cholic acid, bilirubin and phospholipid were estimated in Extracts 1, 3 and 5. Calcium and cholic acid contents were measured in Extracts 2 and 4 . The final residue was weighed after desiccation. Chemical analysis of the final residue was not carried out in the present study.

The following methods were used for quantitative measurement of each component. Fatty acid: a modification of Trout's method using Dole's solution for titration.7 Cholesterol: the method by Leffler and McDougald using ferric chloride. ${ }^{8}$ Cholic acid: the method by Irvin et al. uing furfural.9 Bilirubin: the method of Malloy and Evelyn.10 Phospholipid: the phosphorus content obtained by Bartlett's method ${ }^{11}$ was multiplied by 25. Calcium: titration method using potassium permanganate. ${ }^{12}$

\section{2) Analysis by gas chromatography and infrared absorption spectrum}

Fatty acid composition of each fraction of chloroform extract was studied by these methods. Three to five $\mathrm{mg}$ of each sample were analyzed for fatty acid by gas chromatograph (Hitachi-Perkin Elmer type F-6) after esterification with hydrochloric methanol according to the method of Stoffel et al. ${ }^{13}$ Samples were passed through a $2 \mathrm{~m}$ long column filled with $6 \%$ polyethylene glycol succinate on Chromosorb $\mathrm{W}$ at $210^{\circ} \mathrm{C}$ under a nitrogen pressure of $1.5 \mathrm{~kg} / \mathrm{cm}^{2}$. For identification of fatty acids, methylesters of palmitic, stearic, oleic and 


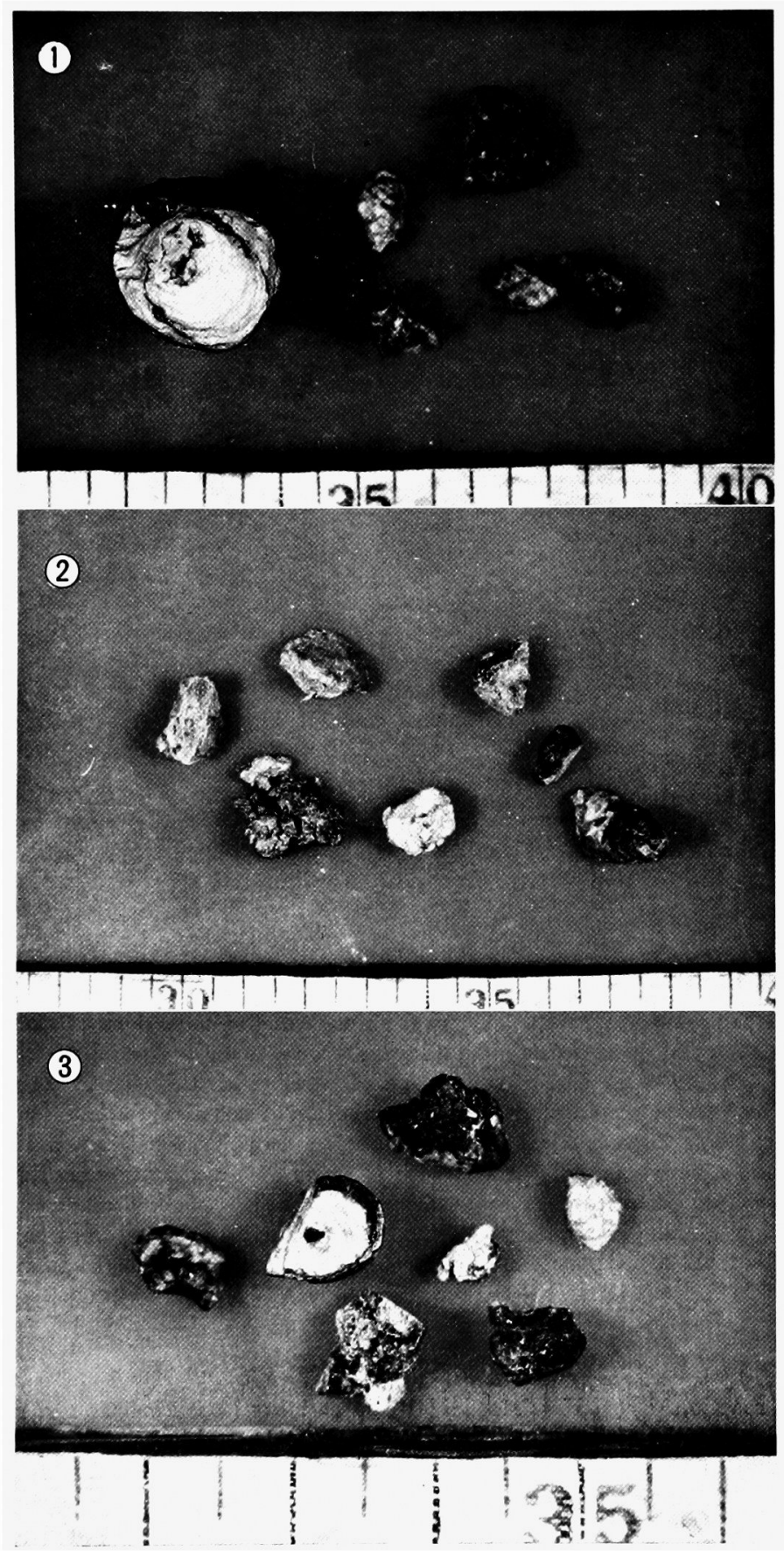

Fig. 1. Fatty acid-calcium stones of sample I.

Fig. 2. Fatty acid-calcium stones of sample II.

Fig. 3. Fatty acid-calcium stones of sample III. 


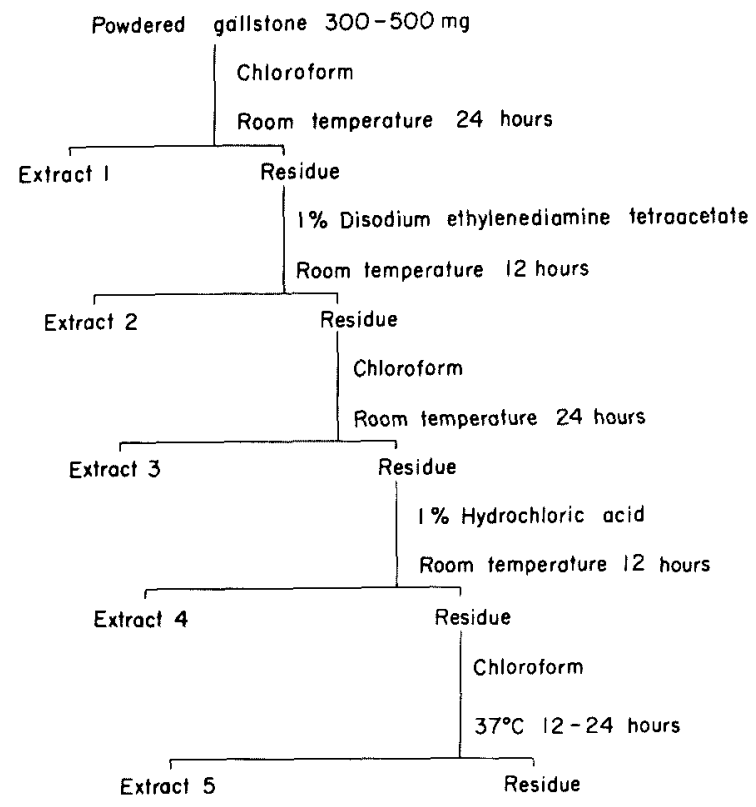

Fig. 4. Method of chemical analysis.

linoleic acids were prepared using the product of these acids manufactured by Sigma Co. and used as the reference standard samples. The peaks of other fatty acids were identified by calculating the relative retention times. For infrared spectrum analysis, Hitachi spectrophotometer (type EPI-S2) and $\mathrm{KBr}$ tablet method ${ }^{14}$ were employed.

\section{3) Histochemical and mineralogical examination}

Good shaped stones were picked up from each of the collections of stones of the 3 patients and 15 to 20 micron thick serial sections were made according to the method by Nakamura. ${ }^{15}$ Each section was placed on a slide glass with the side of resin membrane upwards and examined for morphology and distribution of fatty acid-calcium crystals under polarized light. For histochemical examination of mucopolysaccharides in the gallstone, the sections were placed on slide glasses with the side of resin membrane downwards, so that the cut surface of the stones could be exposed and fixed in formalin vapor. After bleaching with weak alkaline solution of $3 \%$ potassium ferricyanide and washing, the sections were stained by the following methods. Bleaching was controlled under the microscope. For sections 15 to 20 microns thick, satisfactory bleaching was obtained within 30 minutes.

\section{Methods of staining for histochemical examination}

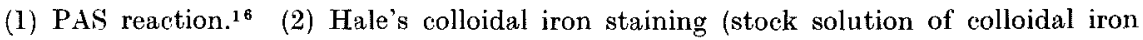
was prepared by Müller-Mowry's method). ${ }^{17}$ (3) $1 \%$ Alcian blue BGX of pH 2.4.17 (4) concentrated $\mathrm{HCl}$ was added to $0.1 \%$ Alcian blue $8 \mathrm{GX}$ to obtain $\mathrm{pHs}$ of $0.5,1.0,1.5,2.0$ and 2.5. (5) $0.5 \%$ Toluidine blue (Pearse's method) ${ }^{18}$ (6) $0.05 \%$ Toluidine blue of which $\mathrm{pH}$ was adjusted to $2.5,4.1$ and 7.0 with citric acid and sodium phosphate (dibasic). (7) $0.05 \%$ Alcian blue containing $0.4 \mathrm{M}$ or $0.9 \mathrm{M}$ magnesium chloride $(\mathrm{pH} 5.8) .^{18,19}$ After staining and washing, the slides were mounted with glycerin-gelatin or glycerin-PV $\mathbf{P}^{\mathbf{2 0}}$ for the microscopic study. 


\section{RESUlTS}

\section{1) Results of chemical analysis}

As is shown in Table 1, the total fatty acid content was $166 \mathrm{mg}$ in $300 \mathrm{mg}$ of pulverized gallstone $(55.3 \%)$ in sample I (Sp. I), $360 \mathrm{mg}$ in $500 \mathrm{mg}(72.0 \%)$ in sample II (Sp. II) and $143 \mathrm{mg}$ in $300 \mathrm{mg}(47.7 \%$ ) in sample III (Sp. III), namely approximately $50 \%$ or more in all samples. The cholesterol contents were $10.3 \%$ in Sp. I, $5.0 \%$ in Sp. II and $22.7 \%$ in Sp. III, i.e., the Sp. III contained a.

TABLE 1. Analytical data of the fatty acid-calcium stones

\begin{tabular}{|c|c|c|c|c|c|c|}
\hline \multirow[b]{3}{*}{ Total fatty acid } & \multicolumn{2}{|c|}{ Sp. I (300 mg) } & \multicolumn{2}{|c|}{ Sp. II (500 mg) } & \multicolumn{2}{|c|}{ Sp. III $(300 \mathrm{mg})$} \\
\hline & $\mathrm{mg}$ & $\%$ & $\mathrm{mg}$ & $\%$ & $\mathrm{mg}$ & $\%$ \\
\hline & 166 & 55.3 & 360 & 72.0 & 143 & 47.7 \\
\hline Fatty acid* & 30 & 10.0 & 26 & 5.2 & 11 & 3.7 \\
\hline Cholesterol & 31 & 10.3 & 25 & 5.0 & 68 & 22.7 \\
\hline Cholic acid & 1.2 & 0.4 & 2.5 & 0.5 & 1.4 & 0.5 \\
\hline Phospholipids & Trace & - & Trace & - & 1.2 & 0.4 \\
\hline Bilirubin & 1.3 & 0.4 & Trace & - & 0.7 & 0.2 \\
\hline Calcium & 11.4 & 3.8 & 25.1 & 5.0 & 12.2 & 4. 1 \\
\hline Residue & 60 & 20.0 & 65 & 13.0 & 48 & 16.0 \\
\hline Total & 270.9 & 90.3 & 477.6 & 95.5 & 274.5 & 91.5 \\
\hline
\end{tabular}

* Fatty acid of extract $I$, which was not combined with calcium.

considerably larger amount of cholesterol than other 2 samples. The cholic acid contents were almost equal in all samples, i.e., $0.4 \%, 0.5 \%$ and $0.5 \%$ in 3 samples, respectively. The calcium contents were $3.8 \%, 5.0 \%$ and $4.1 \%$ in weight in Sps. I, II and III. The ratios in weight of calcium to the fatty acid (as palmitic acid) which is considered to be combined with calcium were $11.9,13.3$ and 10.8 in respective samples, and the molar ratio was about 1:2. Phospholipids and bilirubin were found only in minute amounts. The final residues were $20 \%, 13 \%$ and $16 \%$ in 3 samples, respectively; and the total recoveries including the final residues were $90.3 \%, 95.5 \%$ and $91.5 \%$, respectively.

\section{2) Results of infrared spectrum analysis}

The infrared spectra of the powder of stones of 3 cases are shown in Fig. 5. There are absorption bands of alkan group at $2,930 \mathrm{~cm}^{-1}, 2,850 \mathrm{~cm}^{-1}$ and strong absorption bands at $1,580 \mathrm{~cm}^{-1}, 1,545 \mathrm{~cm}^{-1}$ and $1,474 \mathrm{~cm}^{-1}$, absorption bands at $1,434 \mathrm{~cm}^{-1}, 1,420 \mathrm{~cm}^{-1}, 1,109 \mathrm{~cm}^{-1}$, and $720 \mathrm{~cm}^{-1}$ and equidistant narrow absorption bands between $1,350 \mathrm{~cm}^{-1}$ and $1,180 \mathrm{~cm}^{-1}$. This spectrum is identical with that of calcium palmitate synthesized in vitro, indicating that the main component of the stone was calcium palmitate. In Fig. 6 were shown infrared spectra of fractions of chloroform extracts obtained after treatment of powder of stones with $1 \% \mathrm{HCl}$. The absorption bands at $3,000 \mathrm{~cm}^{-1}, 2,500 \mathrm{~cm}^{-1}, 1,700 \mathrm{~cm}^{-1}, 1,435 \mathrm{~cm}^{-1}, 1,295 \mathrm{~cm}^{-1}$ and $940 \mathrm{~cm}^{-1}$ are those of carboxyl radicals and the bands at $2,930 \mathrm{~cm}^{-1}, 2,860 \mathrm{~cm}^{-1}$, $1,465 \mathrm{~cm}^{-1}$ and $1,412 \mathrm{~cm}^{-1}$ and $1,350 \mathrm{~cm}^{-1}$ are those characteristic of the alkan group. 


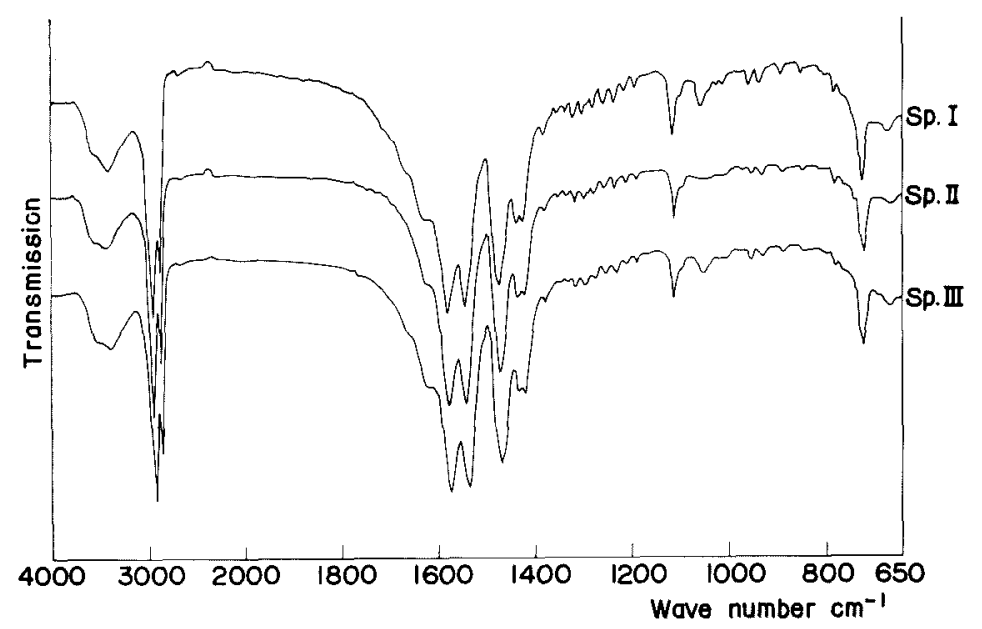

Fig. 5. Infrared spectrua of the powder of the stones. Sps. I, II and III represent samples I, II and III, respectively ( $\mathrm{KBr}$ tablet method).

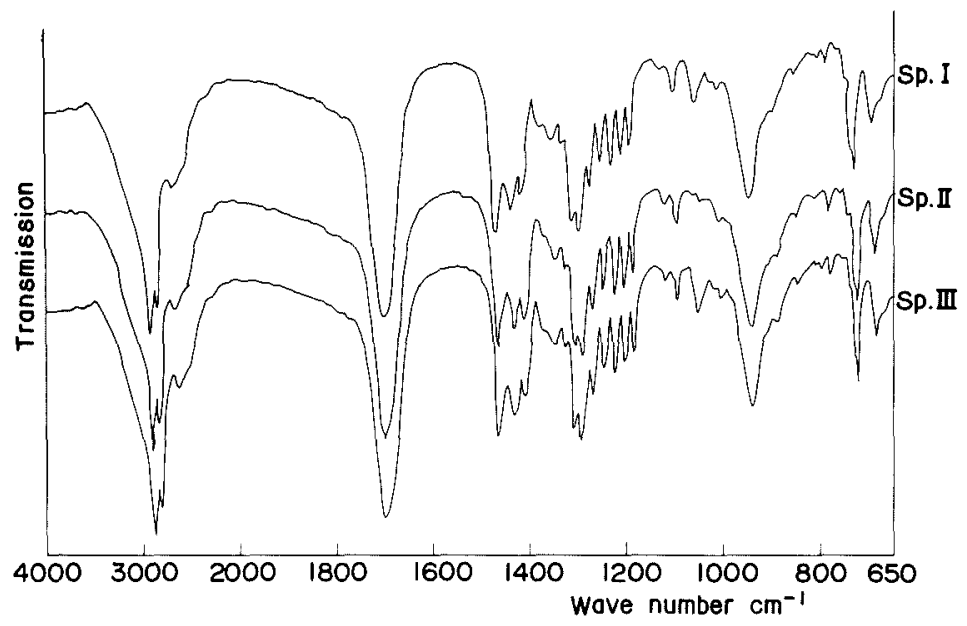

Fig. 6. Infrared spectra of chloroform extracted fractions.

Equidistant small bands between $1,350 \mathrm{~cm}^{-1}$ and $1,180 \mathrm{~cm}^{-1}$ are due to wagging of methyl chains and the bands at $730 \mathrm{~cm}^{-1}$ and $720 \mathrm{~cm}^{-1}$ were ascribed to rocking of methyl chains. The spectra were therefore interpreted as showing that the major component of the extract was palmitic acid.

3) Results of gas chromatographic analysis

As is shown in Fig. 7, it was evident that palmitic acid was predominant in all of the 3 samples. Myristic, pentadecanoic, stearic and oleic acids were also detected in small amounts. The proportion of palmitic acid as is shown in Table 2 was calculated to be $94.8 \% 75.0 \%$ and $83.0 \%$ in 3 samples, respectively. 

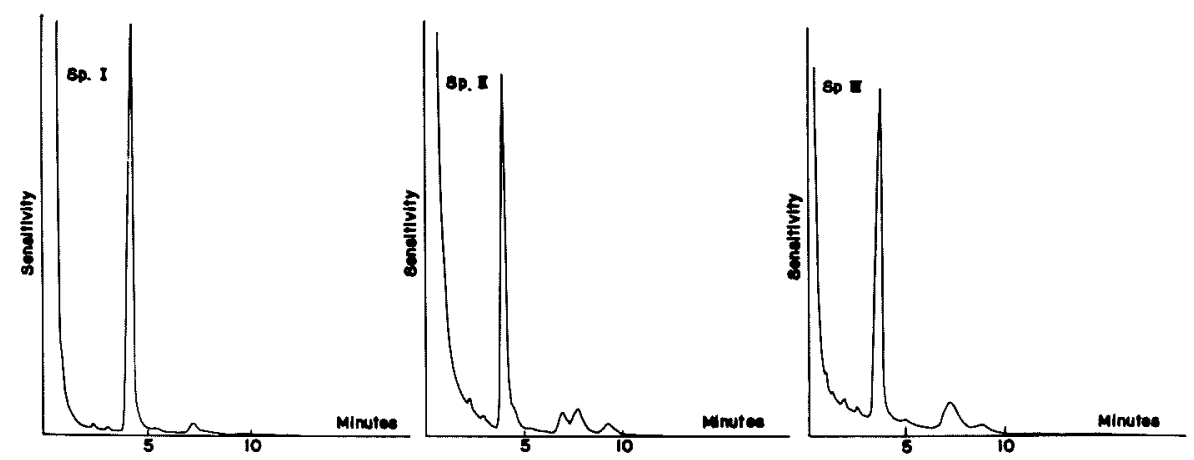

Fig. 7. Gas chromatograms of fatty acids in chloroform extracted fractions (methylester method). It is evident that palmitic acid is predominant in all the 3 samples.

TABLE 2. Fatty acid compsition (in percentage) of samples $I, I I$ and $I I I$

\begin{tabular}{c|c|c|c}
\hline & Sp. I & Sp. II & Sp. III \\
C14:0 & 0.6 & 1.0 & 1.4 \\
C15:0 & 0.5 & 0.6 & 0.8 \\
C16:0 & 94.8 & 75.0 & 83.0 \\
C16:1 & 0 & 0.5 & 0 \\
*C17:0 & 0.4 & Trace & 0.5 \\
C18:0 & 3.8 & 8.1 & 13.1 \\
C18:1 & 0 & 10.6 & 1.2 \\
C18:2 & 0 & 4.8 & 0 \\
* Tentative identification & & &
\end{tabular}

\section{4) The structure of fatty acid-calcium stones}

Under low magnification, the cut surface of the fatty acid-calcium stone showed a laminated structures (Fig. 8). The layers were not concentrically arranged as in calcium bilirubinate stones, but showed an eccentric center giving a characteristic appearance. The laminated structure was rather clear in the periphery than in the central portion of the stones. The layers were made up of bile pigment. Bile pigment was also present in minute amount in the spaces between the crystals of fatty acid-calcium that were present between layers. Observed under polarized light, the crystals of fatty acid-calcium showed a regular fan-shaped arrangement extending from inner to outer layers as is presented in Fig. 9.

\section{5) Results of histochemical examination}

When the sections of the stones were stained after washing with PAS, colloidal iron and Alcian blue, the entire surface of the sections showed a positive reaction. The staining was relatively weak with PAS but was strongly positive with colloidal iron and Alcian blue. In Alcian blue staining, the reaction was more strongly positive for lower $\mathrm{pH}$. Gamma-metachromasia was evident in toluidine blue staining (Pearse's method). In Scott's Alcian blue staining, the reaction was positive when 


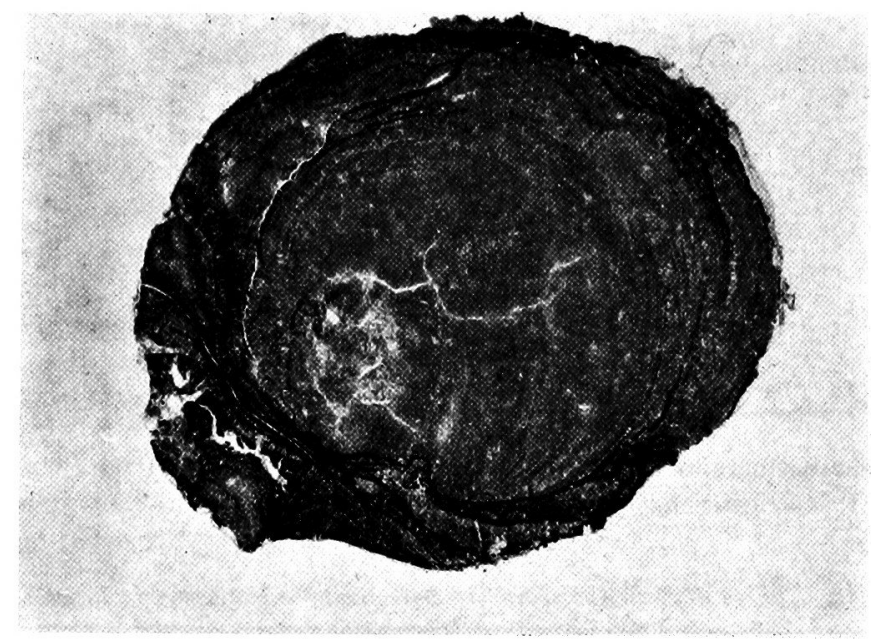

Fig. 8. Cut surface of a fatty acid-calcium stone. Characteristic laminated structure is evident.

$0.4 \mathrm{M} \mathrm{MgCl}_{2}$ solution was used, but was negative when $0.9 \mathrm{M} \mathrm{MgCl}_{2}$ solution was used (Figs. 10 and 11).

The results of these staining reactions suggested the presence of acid mucopolysaccharides, especially sulfated mucopolysaccharides as well as neutral and weak acid mucopolysaccharides in fatty acid-calcium stones. When stained slides were examined in comparison with unstained slides, it was demonstrated that acid mucopolysaccharide was present forming networks in the layers of bile pigment and in spaces between crystals of fatty acid-calcium.

\section{Discussion}

Nishimura described fatty acid-calcium gallstones as dark brown in surface with yellowish-white cut surface with waxy luster, which showed striation as well as laminated structure of eccentrically arranged layers. All samples of the gallstones obtained at our clinic were dark brown in color. They differed from those described by Nishimura, however, in that their cut surface showed a laminated structure consisting of layers of different curvature with an eccentric center, which gave a characteristic feature to it.

Various methods have been devised for chemical analysis of gallstones, and there are certain advantages as well as disadvantages in each of them. In the present study, the method advocated by Nishimura ${ }^{1}$ and $S_{a t 0^{21}}$ was employed. It was found, however, that extraction with ethanol gave a low yield of fatty acid. It was also noted that heating for long time could result in decomposition of fatty acids. Furthermore, ethanol extract of samples treated with $20 \% \mathrm{HCl}$ gave a dark brownish discoloration possibly owing to chemical changes of bilirubin. For these reasons, it 
was considered most appropriate to carry out extraction at a room temperature or a temperature below $37^{\circ} \mathrm{C}$. The fatty acid contents in samples I, II and III were $55.3 \%, 72.0 \%$ and $47.7 \%$, respectively. These values were lower, except in sample II, than those between 64.46 and $81.10 \%$ obtained by Nishimura ${ }^{1,3,4}$ with his samples. Yet it was evident that fatty acid was the major component of the stones. Nishimura noted palmitic acid to be the major component of the fatty acid present in the stone. This was also the case, even though stearic acid was also present in a minute amount in all of our samples. Gallstones containing stearic acid have been reported by Chihara et al. ${ }^{22}$ who used the method of infrared spectrum analysis.

The cholesterol contents in our samples varied from 5.0 to $22.7 \%$ and were a little higher than those reported by Nishimura who obtained the values of 4.10 to $14.30 \%$. Cholic acid and phospholipid were found ony in minute amounts. The bilirubin contents in our samples were considerably lower than those in Nishinura's samples which ranget from $2.26 \%$ to $9.92 \%$. The difference seems to be due to the fact that in the present study the bilirubin content of the final residue was not taken into account. It is likely that the final residue also contained protein, mucopolysaccharide and inorganic ions.

It is known that the gallstones containing a relatively large amount of fatty acids are usually found in the common bile duct or hepatic ducts and that the stones existing within the gallbladder do not contain fatty acids. This fact seems relevant for elucidating the mechanism of formation of fatty acid-calcium stones. Cholelithiasis of this type is usually accompanied by severe infection of the biliary tract, and its clinical manifestations are similar to those due to calcium bilirubinate stones. It would seem, therefore, that $E$. coli infection of the biliary tract is an important factor in the formation of fatty acid-calcium stones.

Fatty acids are present in the bile for the most part as the side chains of phospholipids and neutral fats. It has been revealed that the largest proportion of fatty acid is attached to lecithin in the phospholipid. ${ }^{23}$ Consequently, a mechanism has to be postulated by which fatty acids are split off from lecithin. The possible mechanism has been investigated by Miyake et al. ${ }^{24}$ He found that two factors, exotoxin of $\mathrm{Cl}$. welchii and the pancreatic enzyme that was brought into contact with bile as the result of the reflux of pancreatic juice played an important role in releasing fatty acids into the bile. Of the two factors, he attached a greater importance to the first one. It has been reported, however, that exotoxin of $C l$. welchii contains only phospholipase D. ${ }^{25}$ This enzyme is known to dissociate lecithin into diglyceride and phosphoryl choline. Therefore, the release of saturated fatty acids such as palmitic acid cannot be attributed to the action of this enzyme alone. It is also known that fatty acids are attached to lecithin at its $\alpha$-position. ${ }^{26}$ For fatty acids to be split off, therefore, enzymes that attack $\alpha$-position must be in action. Since a variety of lipases as well as phospholipases are contained in the pancreatic juice, reflux of the pancreatic juice into the bile duct seems to play a more important role. It may be assumed that fatty acids thus released precipitate with calcium increased in the bile as a result of infection. 
It had not been clear what kind of substances in the bile are turned into concrements, until Maki and Suzuki ${ }^{27}$ studied this problem in 1964. They succeeded in obtaining concrement-like aggregates in vitro by adding linear polymer electrolytes such as sodium alginate to suspension of synthesized calcium bilirubinate or calcium carbonate and by delivering kinetic energy to the mixture. On the basis of this result, they concluded that the following three factors are essential for in vivo aggregation and solidification of colloidal substances: condensation of ionic atmosphere and neutralization of $\zeta$-potential due to interaction of organic and inorganic electrolytes, bridging action between colloidal particles by polymer electrolytes, and the effect of kinetic energy.

Nakamura ${ }^{15,29,30}$ developed a new technic for making serial sections of gallstone. Using this technic, he studied from the crystalological and histochemical points of view calcium carbonate stones, calcium bilirubinate stones and gallstone-like concrements synthesized artificially. He found that acid mucopolysaccharides were forming net-works in these stones in such a way as to bridge each crystal particle together. This finding led him to conclude that acid mucopolysaccharides were an important factor in concrement formation of bile components. Matsushiro and Nemoto ${ }^{31}$ carried out chemical analysis of human calcium carbonate stones and found acid mucopolysaccharides including uronic acid, sialic acid and sulfated radicals in the extract. It is reasonable to assume also in fatty acid-calcium stones that acid mucopolysaccharids ere playing a similar role. The findings of the histochemical study of sections of fatty acid-calcium stones were in accord with the assumption. Namely, it was observed that the entire cut surface of these stones gave positive staining reactions for acid mucopolysaccharides and that, in more detailed examinations, acid mucopolysaccharide was present within the layers and in the spaces between fatty acid-calcium crystals forming net-works surrounding crystals. This feature was, in minute detail, quite similar to that observed in calcium carbonate and calcium bilirubinate stones, indicating that acid mucopolysaccharides also played an important role in bringing crystals together in the formation of stones of this type. The crystals appeared, as in cholesterol stones, in needle- or rod-like shapes. Under polarized light, these crystals were seen to extend in a fan-shape outwards from each layer. This finding suggests that the crystals accumulate layer upon layer in the course of time.

On the basis of these findings it can be concluded that fatty acid-calcium stones are, so far as the mechanism of their formation is concerned, similar to calcium bilirubinate stones in that they are produced under the effect of infection, as a result of precipitation of component materials released by enzymes, and that the presence of acid mucopolysaccharides is required for their aggregation and solidification. They appear, however, more like cholesterol stones than calcium bilirubinate stones or calcium carbonate stones, as far as the pattern of arrangement of fatty acid-calcium crystals is concerned. 


\section{References}

1) Nishimura, M. U̇ber die chemische Zusammensetzung der Gallensteine. J. Biochem., 1938, 28, 265-292.

2) Miyake, H., Kuwatsuka, T., Tamesue, N., Nakayama, K., Izumi, H., Yamamoto, H., Aono, K., Oishi, N. \& Hara, A. Comparative studies on the chemical compasition of gallstones in Japan, China and United States. Fukuoka Igaku Zassi(Jap.), 1963, $54,781-826$.

3) Nishimura, M. About fatty acid-calcium stones. Rinsho to Kenkyu (Jap.), 1949, 26, $560-561$.

4) Nishimura, M. Structure and classification of gallstone. Rinsho Geka (Jap.), 1948, 3, 145-151.

5) Maki, T. Pathogenesis of calcium bilirubinate gallstone: Role of E. coli, $\beta$. glucuronidase and coagulation by inorganc ions, polyelectrolytes and agitation. Ann. Surg., 1966, 164, 90-100.

6) Maki, T. \& Suzuki, N. Experimental study on the mechanism of formation of calcium carbonate stones. Tohoku J. exp. Med., 1966, 88, 181-193.

7) Trout, D.L., Estes, E.H.,Jr. \& Friedberg, S.J. Titration of free fatty acids of plasma: A study of current methods and a new modification. J. Lipid Res., 1960, 1, 199-202.

8) Leffler, H.H. \& MeDougald, C.H. Estimation of cholesterol in serum. Amer. $J$. clin. Path., 1963, 39, 311-315.

9) Irvin, J.L., Johnston, C.G. \& Kopala, J. A photometric method for the determination of cholates in bile and blood. J. biol. Chem., 1944, 153, 439-457.

10) Malloy, H.T. \& Evelyn, K.A. The determination of bilirubin with the photoelectric colorimeter. J. biol. Chem., 1937, 119, 481-490.

11) Bartlett, G.R. Phosphorus assay in column chromatography. J. biol. Chem., 1959, 234, 466-468.

12) Clark, E.P. \& Collip, J.B. A study of the Tisdall method for the determination of blood serum calcium with a suggested modification. J. biol. Chem., 1925, 63, 461464.

13) Stoffel, W., Chu, F. \& Ahrens, E.H., Jr. Analysis of long-chain fatty acids by gas-liquid chromatography. Anal. Chem., 1959, 31, 307-308.

14) Nakanishi, K. Infrared Absorption Spectroscopy. Nankodo, Tokyo, 1962.

15) Nakamura, N. Resin-reinforcing technique for sectioning gallstones. Tohoku $J$. exp. Med., 1966, 90, 347-354.

16) Pearse, A.G.E. Histochemistry, Theoretical and Applied, 2nd ed., Churchill, London, 1960.

17) McManus, J.F.A. \& Mowry, R.W. Staining Method, Histologic and Histochemical, Paul B. Hoeber, New York, 1960.

18) Scott, J.E. \& Dorling, J. Differential staining of acid glycosaminoglycans (mucopolysaccharides) by Alcian blue in salt solutions. Histochemie, 1965, 5, 221-233.

19) Stockwell, R.A. \& Scott, J.E. Observations on the acid glycosaminoglycan (muco. polysaccharide) content of the matrix of aging cartilage. Ann, rheum. Dis., 1965 , $24,341-350$.

20) Burstone, M.S. Polyvinyl pyrrolidone as a mounting medium for stains for fat and for azo-dye procedures. Amer. J. clin. Path., 1959, 28, 429-430.

21) Sato, T. Chemical analysis of gallstone. Report 1. On the fundamental experiments of the fixed quantity of gallstone constituents. Hirosaki Igaku (Jap.), 1961, 12, 695706.

22) Chihara, G., Kobayashi, E., Mizushima, A., Shirakuma, K. \& Kameda, H. Medical and biochemical application of infrared absorption spectra. Chem. pharm. Bull., $1960,9,771-777$.

23) Blomstrand, R. Analysis of human bile lipids by gas-liquid chromatography. Acta chem. scand., 1960, 14, 1006-1010.

24) Miyake, H., Kuwazuka, J., Nakamura, Y., Matsuo, H., Tamesue, N., Kodama, M., 
Ozasa, T., Murakoshi, T. \& Yoshimuta, N. About mechanism of gallstone formation -- with special reference to Cl. Welchii. Saishin Igaku (Jap.), 1959, 14, 3088-3102.

25) Bloch, K. Lipid Metabolism. John Wiley and Sons Inc., New York, 1960.

26) Hanahan, D.J., Brockerhoff, H. \& Barron, E.J. The site of attack of phospholipase (lecithinase) A on lecithin: A re-evaluation. J. biol. Chem., 1960, 235, 1917-1923.

27) Maki, T. \& Suzuki, N. On mechanism of coagulation and solidification of gallstone ingredient in bile. Tohoku $J$. exp. Med., 1964, 84, 259-273.

28) Maki, T., Sato, T. \& Suzuki, N. On in vitro synthesis of calcium bilirubinate. Tohoku J. exp. Med., 1964, 82, 117-123.

29) Nakamura, N. Mucopolysaccharides and their significance in gallstone formation. Tohoku J. exp. Med., 1967, 93, 235-247.

30) Maki, T. \& Nakamura, N. Histochemical study on mucopolysaccharides in calcium bilirubinate gallstones. Tohoku J. exp. Med., 1969, 98, 81-86.

31) Matsushiro, T. \& Nemoto, T. Biochemical studies on mucopolysaccharides in calcium carbonate stones. Tohoku J. exp. Med., 1968, 94, 397-406.

Fig. 9. Microphotograph of an unstained section. $15 \mu . \times 200$.

Fatty acid-calcium crystals forming fan-shaped radiation extending outwards are demonstrated.

Fig. 10. Microphotograph of a section stained with $0.1 \%$ Alcian blue (pH 1.5). $15 \mu . \quad \times 40$. Acid mucopolysaccharide (mainly sulfated mucopolysaccharide) is distributed within layer and in the spaces between crystals just like binding fatty acid-calcium crystals together.

Fig. 1I. The same section as Fig. 10 under higher magnification. 15 $\mu . \times 200$. 

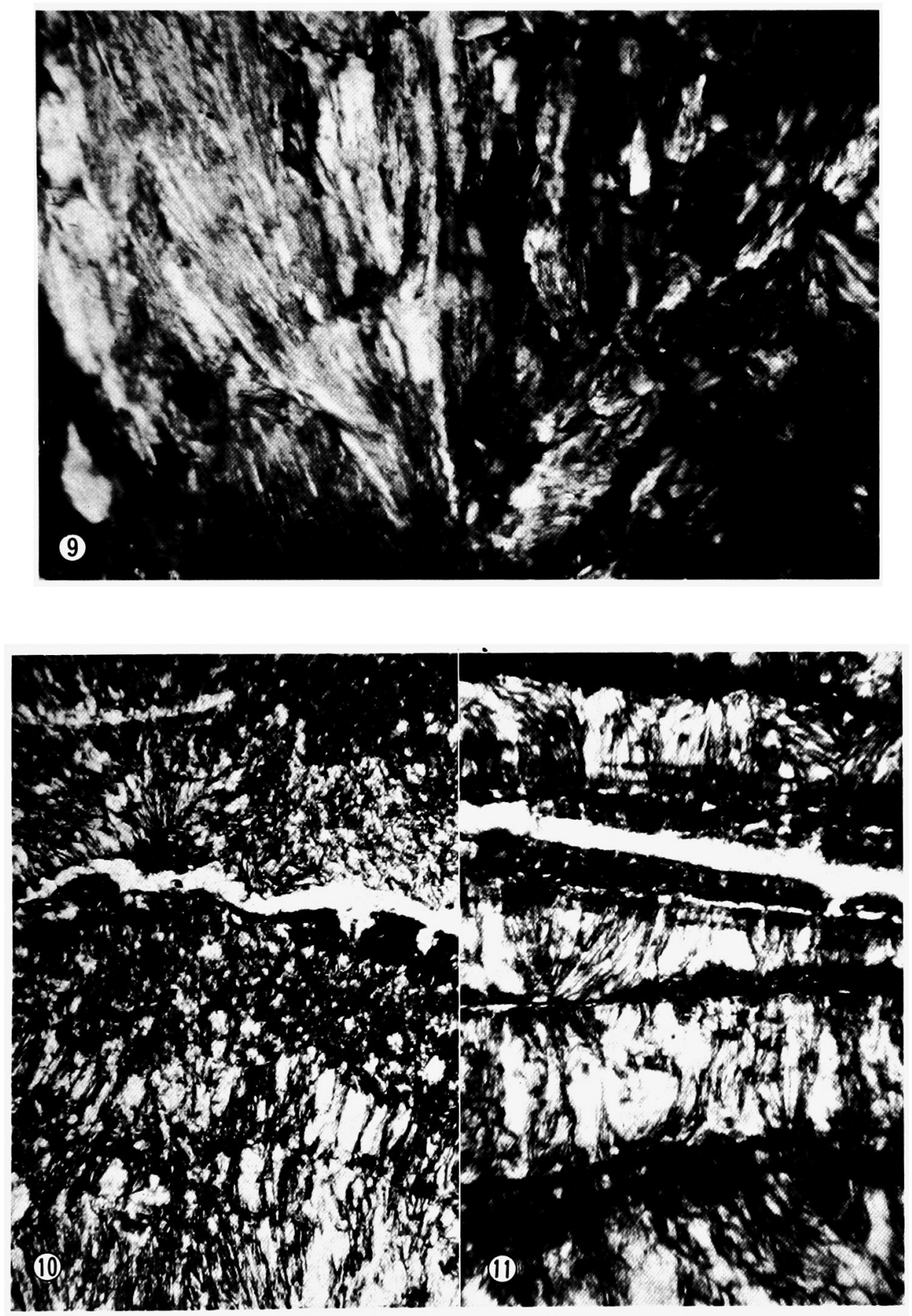